\title{
Mobile learning multidispositivo en la enseñanza universitaria mediante el uso de aplicaciones Web. Experiencia en la asignatura Dirección de la Producción.
}

\author{
Mobile learning multidevice in the university education with Web \\ apps. Experience in the Production Management subject. \\ Francisco David de la Peña Esteban (franciscodavid.delapena@udima.es) \\ María Concepción Burgos García (concepcion.burgos@udima.es) \\ Universidad a Distancia de Madrid, Udima (España)
}

\author{
María Antonia Simón Rodríguez (asimorod@uax.es) \\ Universidad Alfonso X el Sabio (España) \\ http://dx.doi.org/10.12795/EDUCADE.2015.106.05
}

\begin{abstract}
RESUMEN: La irrupción de las nuevas tecnologías de la información acaecida estos últimos años, junto con la variedad de dispositivos móviles asociados a ellas, ha hecho que el Mobile Learning evolucione hacia una realidad multidispositivo. De cara a hacerle frente en la enseñanza universitaria, en este trabajo se propone el uso de aplicaciones web (Web Apps) desarrolladas con Diseño Web Adaptable. Se valida la propuesta mediante la realización de una prueba piloto de Web App para la asignatura Dirección de la Producción. De los resultados obtenidos se desprende el éxito que tiene esta iniciativa, tanto para profesores como alumnos, destacando su accesibilidad e inmediatez. Es válida tanto para Tablet/Ipad como Smartphones/lphone, independientemente del sistema operativo utilizado. También se considera una ayuda al aprendizaje tanto para asignaturas teóricas como prácticas.
\end{abstract}

PALABRAS ClAVE: Mobile Learning, Multidispositivo, Web App, Diseño Web Adaptable, enseñanza universitaria.

\begin{abstract}
The inrush of the new information technologies in the last years, together with the variety of mobile devices associated with them, has done that Mobile Learning evolves towards a multidevice reality. In order to face it in the university education, in this work we proposed the use of web applications (Web Apps) developed with Responsive Web Design. The proposal is validated by the pilot test of Web App for the subject of Production Management- The results show the great success, for teachers and students, of this initiative, emphasizing its accessibility and immediacy. It is valid for both Tablet/lpad as Smartphone/lphone, regardless of operating system used. It is also considered an aid to learning for theoretical and practical subjects.
\end{abstract}

KEYWORDS: Mobile Learning, Multidevice, Web App, Responsive Web Design, university education.

Artículo de investigación. Recibido: 16-004-15 - Versión revisada: 24-09-15, Aceptado: 18-12-15

Licencia Creative Commons BY NC ND · 2015 · Asociación Española de Contabilidad y Administración de Empresas - AECA 


\section{INTRODUCCIÓN AL MOBILE LEARNING}

La gran evolución y revolución de las nuevas tecnologías de la información a la que se está asistiendo en estos últimos años es un factor determinante en el devenir del Mobile Learning, también conocido como M-Learning. Muchos autores han dado una definición de este concepto, como "la aplicación de los dispositivos electrónicos móviles para fines educativos" (Brazuelo \& Cacheiro, 2010), "el uso de dispositivos electrónicos móviles y de bolsillo, tales como teléfonos móviles, portátiles, PDAs y tablet PC usados para la formación, aprendizaje y enseñanza" (Sarrab et al., 2012), o "el acceso a través de dispositivos móviles a los contenidos de un curso y todos los materiales de enseñanza respectivos" (Molnár, 2012).

De entre las muchas ventajas que ofrece el Mobile Learning cabe destacar la conectividad mediante dispositivos portátiles, de manera inmediata y desde cualquier sitio (Valero et al., 2012). También tiene otras virtudes, como la posibilidad de ofrecer diversas formas de aprendizaje (Gikas \& Grant, 2013), que es un medio para el aprendizaje colaborativo (Ozuorcun \& Tabak, 2012), y que es ideal para una preparación del estudio "just-in-time", promoviendo la interacción alumno-profesor (Sarrab et al., 2012).

Por otro lado, también existen algunos problemas en su puesta en la práctica, ya que resulta difícil medir el grado en que ha contribuido al aprendizaje (Ozuorcun y Tabak, 2012), y en muchas ocasiones el profesorado es reacio a la implantación de las nuevas tecnologías, estando también presente el problema de las continuas adaptaciones que requiere por tener un corto ciclo de vida (Gikas y Grant, 2013).

La penetración de los dispositivos móviles es cada día mayor, llegando a que el $90 \%$ de los internautas posee un teléfono inteligente, también conocido como smartphone (VI Estudio Anual IAB Spain Mobile Marketing, 2014). Las velocidades de conexión por redes móviles han llegado a 5,2 MBPS en España, siendo la media mundial de 4,5 MBPS (Estudio DIGITAL, SOCIAL \% MOBILE IN 2015), lo que proporciona unas infraestructuras más que suficientes para poder utilizar los smartphones para la carga y descarga de contenidos en la nube.

De forma simultánea se observa un significativo crecimiento en el mercado de Tablets /Ipads, (Estudio KPCB Internet Trends, 2014), siendo un complemento perfecto al smartphone para ser usado en Mobile Learning.

La tecnología avanza y la enseñanza debe adaptarse y hacerse atractiva para las nuevas generaciones, siendo cada vez más necesario el uso de aplicaciones (Apps) para dispositivos móviles en la enseñanza universitaria. Es necesario comentar que existen diversos sistemas operativos, destacando Android e iOS de Apple. Esto ocasiona que para que las Apps sean accesibles a todos los estudiantes, primero deben tener un dispositivo móvil, y en segundo lugar esa aplicación debe también haber sido desarrollada para su sistema operativo.

El objetivo del presente trabajo es proponer un nuevo modelo práctico de aplicaciones para Mobile Learning Multidispositivo en la enseñanza universitaria, mediante el uso de Web Apps con Diseño Web Adaptable. Para su propuesta en primer lugar se ha realizado un estudio de la literatura en Mobile Learning en la enseñanza universitaria. A partir de aquí la metodología que se ha seguido en la presente investigación ha sido la siguiente. Se ha analizado el Diseño Web Adaptable, y las ventajas que proporciona en un entorno multidispositivo. Para comprobar su validez se hizo una prueba piloto en una asignatura universitaria a distancia. Por último se hizo un análisis de la Web App mediante la realización de encuestas y entrevistas. 
La estructura que se ha plasmado es la siguiente. En el punto 2 del presente artículo se van a detallar experiencias previas de uso de aplicaciones para dispositivos móviles que existen en la literatura existente. En el punto 3 se detallará la metodología expuesta. Los resultados alcanzados a través de ella se mostrarán en el punto 4. Por último en el punto 5 se expondrán las conclusiones.

\section{APPS EN ENSEÑANZA UNIVERSITARIA}

Dentro de estas Apps, hay dos grupos bien diferenciados. Aquellas que se utilizan como complemento en las propias clases presenciales (Classroom apps), y las que son utilizadas como complemento externo a las clases (Distance learning apps). Este artículo se va a centrar en estas últimas. En el estudio de la literatura existente, se han detectado como más importantes las siguientes experiencias previas:

- Existe una propuesta de un Servicio de Aprendizaje (Yang \& Wang, 2011), con dispositivos móviles Android para los alumnos, y con un sistema basado en web para los profesores. Se buscaba el aprendizaje colaborativo a través de la realización de una serie de tareas. Los progresos de los alumnos podían ser seguidos gracias a su almacenamiento en la nube. Se hizo una prueba piloto del mismo en la Universidad de Tung-Man (Taiwan), siendo los resultados alcanzados muy positivos tanto a nivel de aprendizaje, como de estabilidad y diseño del sistema.

- También se ha realizado un análisis de la importancia de las aplicaciones para el MLearning en el sector educativo (Bidin \& Zidin, 2012). Para su implantación con éxito se debe hacer un estudio de las características que deben tener los dispositivos móviles (usabilidad y características técnicas), las expectativas de los estudiantes (costes y obsolescencia) y las ventajas pedagógicas de su uso (aprendizaje colaborativo, aprendizaje interactivo, blended learning, aprendizaje basado en problemas).

- Otra aproximación se basó en realizar un análisis DAFO a partir de una experiencia en M-Learning con Ipad (Hargis et al., 2013), encontrando las siguientes:

-Debilidades: hay que decir a los estudiantes lo que deben hacer, los profesores que son contrarios a la tecnología, se necesita almacenamiento en red, y la formación a impartir para profesores y alumnos.

-Amenazas: el desalineamiento entre la calificación y la enseñanza, el lento desarrollo de materiales educativos, y que se utilice únicamente para leer.

-Fortalezas: amplio soporte técnico, gran repositorio de apps, aumenta el compromiso de los alumnos, el profesor es visto como un facilitador, el lpad es más fácil de usar que los portátiles, y la duración de su batería es mucho mayor.

-Oportunidades: el desarrollo de contenidos adaptados a la tecnología móvil, y la posibilidad de otorgar calificaciones como por ejemplo con el uso de rúbricas.

- La posibilidad de realizar valoraciones de trabajos de matemáticas universitarios a través de Tablets (Isabwe, Reichert \& Carslsen, 2013). Las valoraciones se hacían entre los propios estudiantes (peer-assessment) con la ayuda de un Entorno de Aprendizaje Virtual (VLE, Virtual Learning Environment) que recopilaba los trabajos y los distribuía entre los alumnos para su valoración. Los estudiantes mostraron su aceptación del método utilizado, pero señalando la necesidad de una herramienta integrada que mejore la usabilidad técnica y pedagógica del sistema.

- El diseño de un Sistema de Aprendizaje Móvil (MLS, Mobile Learning System) en una plataforma Android, incluyendo el desarrollo del software, los requerimientos del sistema y el análisis técnico del mismo (Kong, 2013). 
- El uso de tablets para una asignatura de Marketing (Oberer \& Erkollar, 2013). La tablet es usado para búsqueda de información y seguimiento de las redes sociales, así como para videoconferencias con Google + (Hangout). No se utiliza una app específica para la asignatura, sino que aprovecha los recursos que le proporcionan otras app. Como resultado se resalta que se logró una gran interconexión entre alumnos, y la aplicabilidad de los conceptos de la materia gracias a la tablet.

- Una experiencia con una lección de arte para ipad/ipod y su interacción en un museo (Martin \& Ertzberger, 2013). Realmente el dispositivo se utilizaba como guía para realizar la visita.

- El desarrollo de una aplicación para Android para enseñar cómo se configuran los routers y los switches a través de dispositivos móviles, en la University of Technology Mara (Malaysia). Expusieron los pasos a realizar para el desarrollo de la misma, así como el plan de pruebas a seguir para validarla (Mamat \& Azmat, 2013).

- La propuesta de un modelo teórico de aplicación híbrida para dispositivos móviles, válida para Android y Blackberry (Setiabudi \& Tjahyana, 2013). Se trata de una aplicación web, con diseño adaptable al tamaño de la pantalla, que utiliza el navegador web nativo del dispositivo. Evidenciaron que las características más deseables eran para compartir archivos, recibir notificaciones y la planificación de la asignatura.

- La utilización del lpad como apoyo pedagógico en la clase en un curso universitario de programación en C (Amer \& Ibrahim, 2014). El resultado indica que fue introducido sin apenas esfuerzo, y que los estudiantes lo vieron como un entorno familiar de aprendizaje, facilitaba el trabajo en grupo, la creatividad así como el aprendizaje independiente.

Para el caso concreto de la enseñanza universitaria a distancia:

- La realización de una encuesta tanto a profesores como a estudiantes sobre el aprendizaje con teléfonos móviles en la enseñanza a distancia (Kousar \& Scholar, 2013). Su conclusión es que la tecnología móvil puede ser usada con éxito para facilitar el aprendizaje.

- La propuesta de un modelo práctico de aplicación basándose en las preferencias de los alumnos y en la experiencia piloto realizada en la asignatura de Dirección de Operaciones (de la Peña \& Burgos, 2015). Se desarrolló una aplicación para Android que contenía documentación, vídeos, audios, calendario, envío de emails y llamadas al profesor. Los estudiantes evidenciaron su gran predisposición hacia las apps, y valoraron muy positivamente la experiencia.

TABLA 1. RESUMEN DE LAS EXPERIENCIAS PREVIAS

\begin{tabular}{|l|l|}
\hline \multicolumn{1}{|c|}{ Autores } & \multicolumn{1}{|c|}{ Descripción } \\
\hline $\begin{array}{l}\text { Yang \& Wang, } \\
2011\end{array}$ & $\begin{array}{l}\text { Propuesta de un Servicio de Aprendizaje (Yang \& Wang, 201 1), con } \\
\text { dispositivos móviles Android para los alumnos, y con un sistema } \\
\text { basado en web para los profesores. }\end{array}$ \\
\hline $\begin{array}{l}\text { Bidin \& Zidin, } \\
2012\end{array}$ & $\begin{array}{l}\text { Realizaron un análisis de la importancia de las aplicaciones para el } \\
\text { M-Learning en el sector educativo. }\end{array}$ \\
\hline $\begin{array}{l}\text { Hargis et al., } \\
2013\end{array}$ & $\begin{array}{l}\text { Se hizo un análisis DAFO a partir de una experiencia en M-Learning } \\
\text { con Ipad. }\end{array}$ \\
\hline
\end{tabular}


F. David de la Peña, M. Concepción Burgos, M. Antonia Simón.

Mobile learning multidispositivo en la enseñanza universitaria mediante el uso de aplicaciones Web

\begin{tabular}{|l|l|}
\hline \multicolumn{1}{|c|}{ Autores } & \multicolumn{1}{c|}{ Descripción } \\
\hline $\begin{array}{l}\text { Isabwe, Reichert } \\
\text { \& Carslsen, 2013 }\end{array}$ & $\begin{array}{l}\text { Estudiaron la realización de valoraciones, entre los propios } \\
\text { estudiantes, de trabajos de matemáticas universitarios a través de } \\
\text { Tablets. }\end{array}$ \\
\hline Kong, 2013 & $\begin{array}{l}\text { Expuso el diseño de un Sistema de Aprendizaje Móvil (MLS, Mobile } \\
\text { Learning System) en una plataforma Android. }\end{array}$ \\
\hline $\begin{array}{l}\text { Oberer \& } \\
\text { Erkollar, 2013 }\end{array}$ & $\begin{array}{l}\text { Se realizó el uso de tablets para una asignatura de Marketing, } \\
\text { mediante la utilización de redes sociales y Hangout. }\end{array}$ \\
\hline $\begin{array}{l}\text { Martin \& } \\
\text { Ertzberger, 2013 }\end{array}$ & $\begin{array}{l}\text { Se impartió una lección de arte para ipad/ipod, y su interacción en } \\
\text { un museo. }\end{array}$ \\
\hline $\begin{array}{l}\text { Mamat \& Azmat, } \\
2013\end{array}$ & $\begin{array}{l}\text { Se desarrolló una aplicación para Android para aprender cómo se } \\
\text { configuran los routers y los switches a través de dispositivos móviles. }\end{array}$ \\
\hline $\begin{array}{l}\text { Setiabudi \& } \\
\text { Tjahyana, 2013 }\end{array}$ & $\begin{array}{l}\text { Propusieron un modelo teórico de aplicación híbrida para } \\
\text { dispositivos móviles, válida para Android y Blackberry. }\end{array}$ \\
\hline $\begin{array}{l}\text { Amer \& lbrahim, } \\
2014\end{array}$ & $\begin{array}{l}\text { La utilización del Ipad como apoyo pedagógico en la clase en un } \\
\text { curso universitario de programación en C. }\end{array}$ \\
\hline $\begin{array}{l}\text { Kousar \& } \\
\text { Scholar, 2013 }\end{array}$ & $\begin{array}{l}\text { Es un estudio sobre el aprendizaje con teléfonos móviles en la } \\
\text { enseñanza a distancia. }\end{array}$ \\
\hline $\begin{array}{l}\text { de la Peña \& } \\
\text { Burgos, 2015 }\end{array}$ & $\begin{array}{l}\text { Realizaron la propuesta de un modelo práctico de aplicación } \\
\text { basándose en las preferencias de los alumnos y en la experiencia } \\
\text { piloto realizada. }\end{array}$ \\
\hline
\end{tabular}

Las experiencias que muestra la tabla 1 son válidas para unos determinados tipos de dispositivos. La realidad tecnológica existente es que el entorno actual es multidispositivo, existiendo numerosos dispositivos móviles y sistemas operativos diferentes con los que interactuar. Esto se ha tenido en cuenta en otros artículos, aunque con poca profusión. La creación de una herramienta de autor para crear contenidos interactivos para múltiples dispositivos (Gordillo et al., 2013), y la propuesta de una arquitectura de soporte heterogéneo en entornos de aprendizaje multidispositivo pero aplicada únicamente para las clases presenciales, no para Mobile Learning (Giemza et al., 2012).

Todas estas referencias sirvieron de base para pensar en la necesidad de crear un modelo de aplicación que fuera compatible con todo tipo de dispositivo móvil, e independiente de su sistema operativo, así como para definir los contenidos que sería interesante que tuviera.

\section{DISEÑO DE EXPERIENCIA Y METODOLOGÍA}

La metodología que se ha seguido en la presente investigación ha sido la siguiente. Primero se propone un nuevo modelo práctico de aplicaciones para Mobile Learning Multidevice, mediante el uso de Web Apps con Diseño Web Adaptable. Para comprobar su validez se hizo una prueba piloto en una asignatura universitaria a distancia, donde se procedió al desarrollo y diseño de una Web App como 
complemento al estudio. Por último se hizo un análisis de la Web App mediante una encuesta realizada a los estudiantes de la asignatura, así como la realización de entrevistas personales a estudiantes y profesores de la Facultad de ADE y Economía de la Universidad a Distancia de Madrid, Udima.

\subsection{Nuevo modelo práctico de aplicaciones para Mobile Learning Multidispositivo: Web Apps}

Hoy en día cada estudiante se encuentra ante una experiencia multidispositivo (multidevice). Como ya se ha visto en apartado 1, la mayoría de las personas usa smartphones, tiene en su hogar una tablet o ipad, así como PCs y portátiles. En este nuevo entorno es necesario el poder crear contenidos que sean accesibles desde todos ellos, y que resulten económicos desde el punto de vista de tiempo y recursos utilizados. Para poder lograr esto, en este artículo se propone la creación de Web Apps como complemento al estudio y aprendizaje de los estudiantes. Una Web App puede definirse como una aplicación que se visualiza en los navegadores web, pero que tiene la apariencia de una aplicación desarrollada para dispositivos móviles, disponiendo de un menú de navegación tipo. Las principales ventajas que tiene es que no es necesaria su descarga, y la actualización de sus contenidos se realiza en la nube, sin necesidad de tener que actualizar o volver a instalar la aplicación.

La creación de las Webs Apps se hace mediante el Diseño Web Adaptable (Responsive Web Design - RWD). Este diseño se basa en 3 características técnicas principales (Baturay \& Birtane, 2013):

-El lenguaje de programación utilizado es HTML5, con hojas de estilos CSS3 y el uso de "media queries".

-El contenido de la web se readapta al tamaño y orientación de la pantalla.

-Los contenidos multimedia y las imágenes tienen tamaños flexibles, redimensionándose en función de la pantalla.

Existen otras ventajas del Diseño Web Adaptable (Baturay \& Birtane, 2013). No es necesario el uso de zoom ni de barras de desplazamiento (scroll) laterales, lo que permite una navegación realmente sencilla. También está el hecho de que únicamente se necesita un site o web única, que valdrá tanto para Pcs, portátiles y dispositivos móviles. Utilizando el Diseño Web Adaptable se logra así mismo evitar el problema de la compatibilidad de los distintos dispositivos con la tecnología flash.

La gran mayoría de los dispositivos móviles fabricados hoy en día son compatibles con este tipo de diseño, lo que lo hace ideal para su uso en Mobile Learning Multidevice. Una experiencia de éxito de este tipo de Web App en el ámbito universitario, aunque no como soporte a una asignatura para Mobile Learning, está en la Biblioteca de ciencias de la salud de la University of Maryland (Glassman \& Shen, 2014).

En base a lo expuesto se procedió a desarrollar una Web App para Dirección de la Producción, una asignatura del Grado en Administración y Dirección de Empresas de la Universidad a Distancia de Madrid, Udima. Su objetivo es que sirva de complemento para el aprendizaje de la asignatura. Los módulos elegidos se basan en el estudio realizado por de la Peña y Burgos (2015), y en las posibilidades tecnológicas disponibles. 


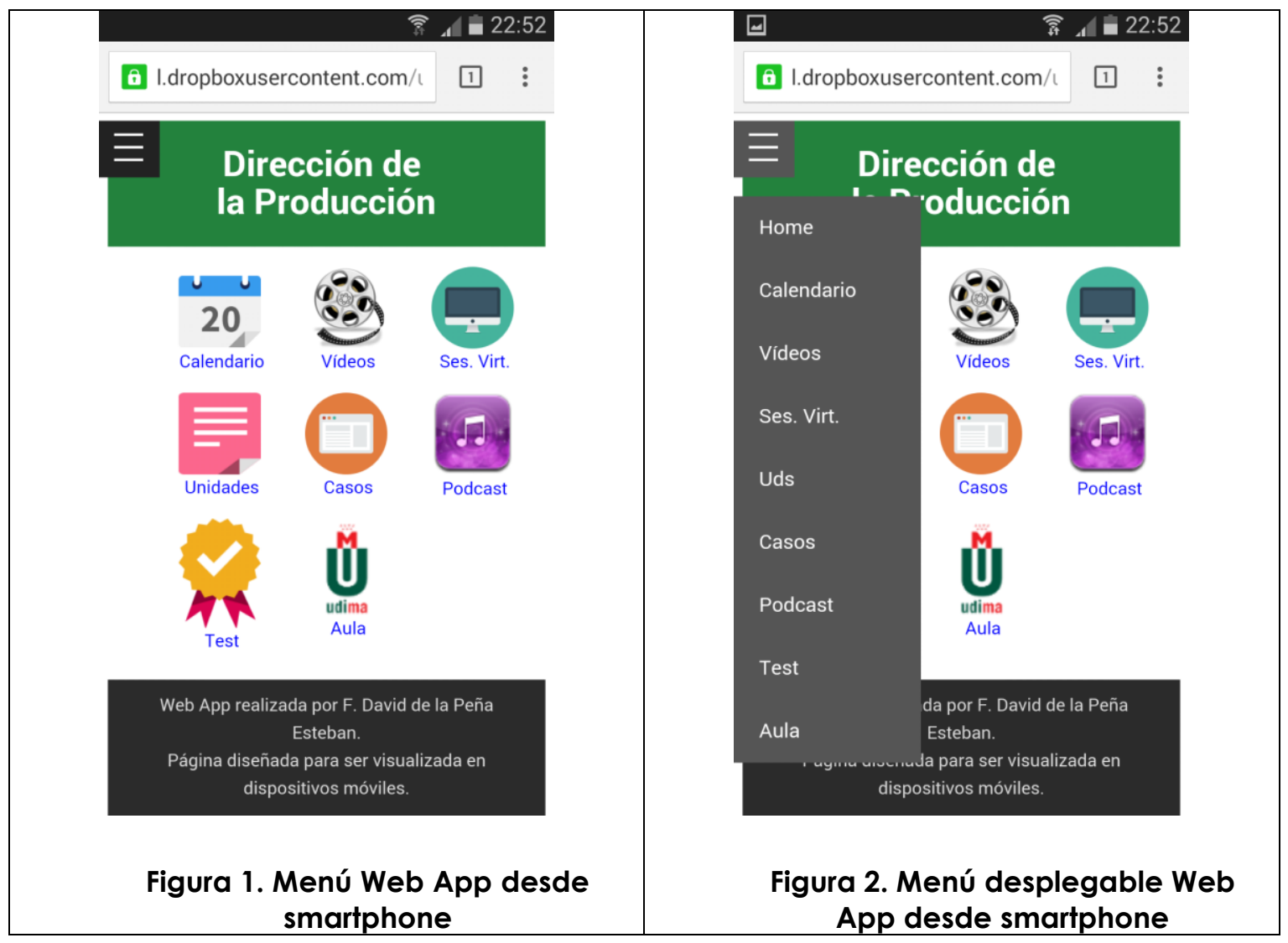

El menú de la Web App, como se observa en las figuras 1 y 2, consta también de un menú desplegable que da acceso a los distintos módulos de la aplicación web. Estos módulos son los siguientes:

-Calendario: muestra las fechas límite para realizar todas las actividades y controles, así como las fechas de las clases virtuales que se realizan en la asignatura.

-Vídeos: están enlazados todos los vídeos, tanto teóricos como prácticos, realizados por el profesor de la asignatura, como se muestra en la figura 3. Al pulsar sobre el enlace, se abre la aplicación de Youtube del dispositivo móvil.

-Sesiones virtuales: desde este icono se accede a la grabación de las clases virtuales celebradas en esta asignatura por el profesor.

-Unidades: se accede al resumen de las distintas unidades de la materia.

-Casos: el estudiante puede elegir estudiar diversos casos prácticos resueltos de la asignatura. En las figuras 4 y 5 se muestran dos de los casos propuestos, desde el punto de vista de un una tablet y un smartphone respectivamente.

-Podcast: existe un listado de audios en formato mp3 que puede escuchar el estudiante de las distintas unidades, tal y como se ve en la figura 6.

-Test: son los cuestionarios de repaso de las unidades de la asignatura, siendo las preguntas de tipo verdadero / falso.

-Aula: es un acceso directo al Aula Moodle de la asignatura. 
F. David de la Peña, M. Concepción Burgos, M. Antonia Simón. Mobile learning multidispositivo en la enseñanza universitaria mediante el uso de aplicaciones Web

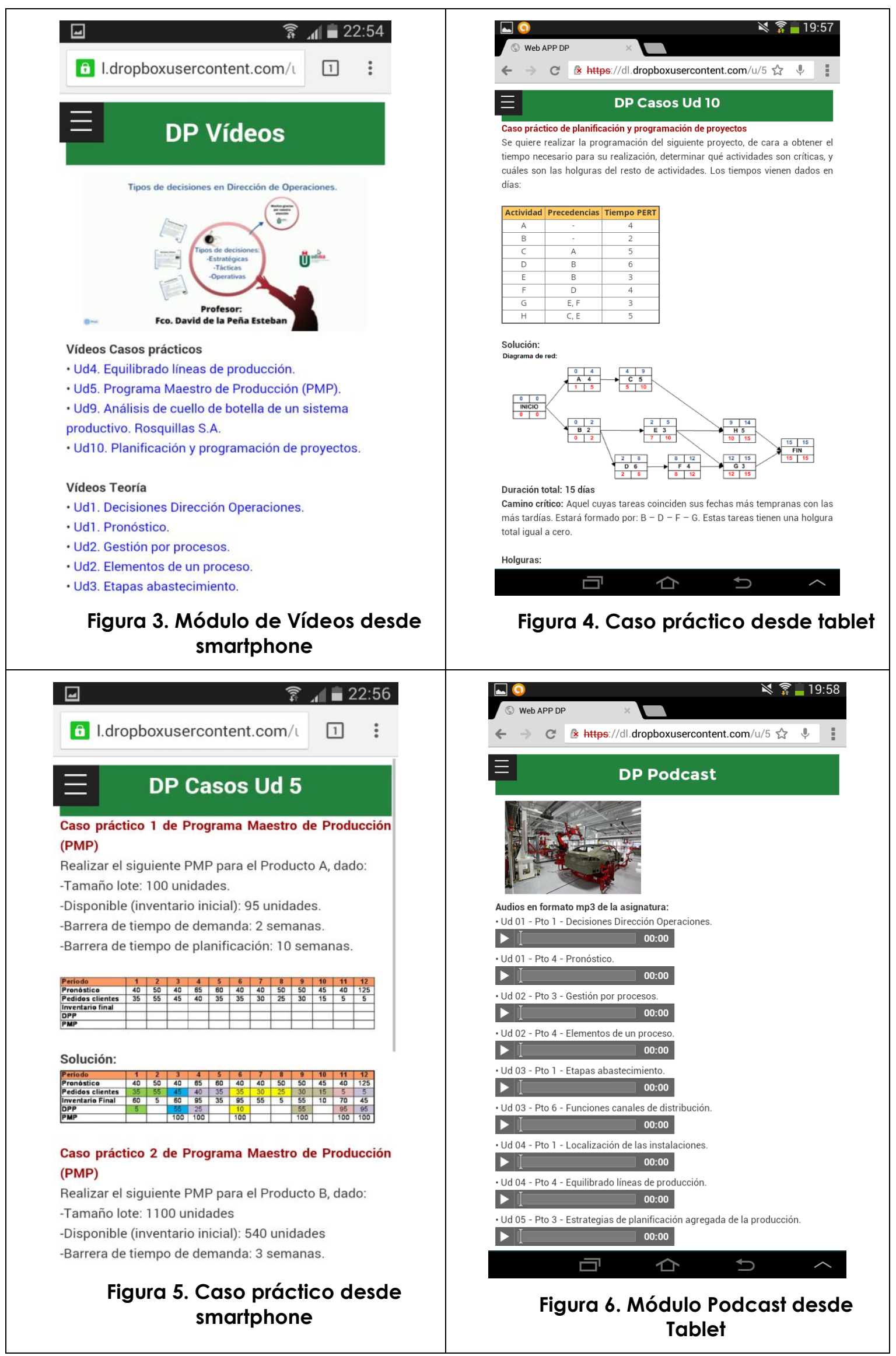




\subsection{Análisis de la Web App.}

La primera parte del análisis se realizó mediante encuesta al alumnado de la asignatura, 33 estudiantes. La Web App estuvo operativa durante un periodo de 2 meses y medio, hasta el día del examen.

La encuesta se realizó mediante un formulario creado en Google Docs (ver anexo 1) a través del que se pretendían analizar los siguientes aspectos generales: utilidad, usabilidad, seguimiento de la asignatura, así como la percepción del estudiante acerca de las aplicaciones y el aprendizaje. La valoración de los estudiantes sobre cada uno de los módulos de la Web App era otro de los objetivos de la encuesta.

También se quería hacer un análisis del uso de la Web App, como el número de visitas realizado, el lugar dónde se encontraba y su tiempo medio.

Por último se quiso conocer en base a la experiencia de los estudiantes, cuál creen que es el mejor dispositivo para su uso, qué ventajas tiene y qué mejoras sería deseable incluir.

Los resultados de la encuesta se detallan en el siguiente punto. En todos aquellos ítems que se valoraban mediante una escala numérica, se utilizó la escala de Likert. Esta escala utiliza 5 niveles de respuesta de 1 (Totalmente desacuerdo) a 5 (Totalmente de acuerdo).

Debido a la insuficiencia en la tasa de respuesta (36\%) y al probable sesgo de la muestra, se procedió a realizar entrevistas en persona, de una duración de 20 a 30 minutos cada una, en las que se pretendía evaluar más cualitativamente este tipo de aplicaciones. Las entrevistas se realizaron a 3 grupos distintos:

-5 alumnos de la asignatura que no habían contestado la encuesta.

-5 alumnos de asignaturas de la Facultad de ADE y Economía que nunca habían visto una aplicación de este estilo.

-5 profesores de la Facultad de ADE y Economía de las siguientes materias: Matemáticas, Economía, Organización de empresas, Dirección Financiera, Habilidades profesionales.

El guión que se utilizó para la entrevista puede verse en el anexo + 2 .

\section{RESULTADOS ALCANZADOS}

\subsection{Encuesta}

De los 33 alumnos, contestaron 12 (36\%). De ellos el 75\% (9), habían utilizado la Web App, mientras que el restante $25 \%$ (3) no la habían usado.

De estos últimos, las razones por la que no la utilizaron, según se observa en la tabla 2 , fueron las siguientes. En primer lugar la falta de predisposición a utilizar su dispositivo móvil para el uso de la Web App, y en segundo término por no disponer de dispositivo móvil. 
F. David de la Peña, M. Concepción Burgos, M. Antonia Simón. Mobile learning multidispositivo en la enseñanza universitaria mediante el uso de aplicaciones Web

tabla 2. Razones no utilización de la Web App

\begin{tabular}{|l|c|c|}
\hline Razón & No respuestas & Porcentaje \\
\hline $\begin{array}{l}\text { Tengo Smartphone / Iphone pero no la iba a } \\
\text { utilizar }\end{array}$ & 1 & $33 \%$ \\
\hline Tengo Tablet/ lpad pero no la iba a utilizar & 1 & $33 \%$ \\
\hline No dispongo de dispositivos móviles & 1 & $33 \%$ \\
\hline
\end{tabular}

\subsubsection{Valoración general.}

Los estudiantes que contestaron que habían utilizado la Web App dieron las siguientes valoraciones (según se observa en la tabla 3): la percepción general es muy positiva con respecto a la utilidad y usabilidad, con unas puntuaciones superiores al 4,4 de media. Destaca el hecho de que están predispuestos a su utilización de una manera más general, con una puntuación media de 4,89. También consideran que con su ayuda han mejorado en el seguimiento de la asignatura, con una puntuación superior a 4,2 .

tabla 3. Valoración general de la Web App

\begin{tabular}{|l|c|c|c|c|}
\hline Valoración general & Mediana & Media & Moda & $\begin{array}{c}\text { Desviación } \\
\text { típica }\end{array}$ \\
\hline Utilidad & 5 & 4,56 & 5 & 0,53 \\
\hline Usabilidad & 5 & 4,44 & 5 & 0,73 \\
\hline $\begin{array}{l}\text { Mi seguimiento de la asignatura ha mejorado } \\
\text { después de descargar la aplicación }\end{array}$ & 4 & 4,22 & 4 & 0,97 \\
\hline $\begin{array}{l}\text { Creo que la existencia de aplicaciones en las } \\
\text { asignaturas mejoraría mi aprendizaje y estaría } \\
\text { dispuesto a descargármelas. }\end{array}$ & 5 & 4,89 & 5 & 0,33 \\
\hline
\end{tabular}

\subsubsection{Valoración módulos.}

La puntuación asociada a cada uno de los módulos fue la: que se observa en la tabla 4. Todos los módulos tienen una alta puntuación, siendo los mejor posicionados los relacionados con vídeos de apoyo, así como con los casos prácticos, con unas puntuaciones medias superiores al 4,4. Por el contrario los menos valorados son los Podcast y el Calendario, con una puntuación media superior a 3,8 .

Tabla 4. Valoración módulos de la Web App

\begin{tabular}{|l|c|c|c|c|}
\hline Valoración módulos & Mediana & Media & Moda & Desviación típica \\
\hline Calendario & 4 & 4,00 & 4 & 1,00 \\
\hline Vídeos Teoría & 4 & 4,44 & 4 & 0,53 \\
\hline Vídeos Casos & 4 & 4,44 & 4 & 0,53 \\
\hline
\end{tabular}


F. David de la Peña, M. Concepción Burgos, M. Antonia Simón.

Mobile learning multidispositivo en la enseñanza universitaria mediante el uso de aplicaciones Web

\begin{tabular}{|l|c|c|c|c|}
\hline Valoración módulos & Mediana & Media & Moda & Desviación típica \\
\hline Sesiones Virtuales & 5 & 4,33 & 5 & 1,00 \\
\hline Unidades & 4 & 4,11 & 4 & 0,93 \\
\hline Casos prácticos & 5 & 4,44 & 5 & 1,01 \\
\hline Podcast & 4 & 3,89 & 4 & 0,78 \\
\hline Test & 5 & 4,11 & 5 & 1,36 \\
\hline Acceso a Aula & 4 & 4,22 & 5 & 0,83 \\
\hline
\end{tabular}

\subsubsection{Análisis de uso.}

Con respecto al número de veces que habían visitado la Web App, la gran mayoría de los estudiantes, el 78\%, habían visitado la Web App como mínimo una vez a la semana. Solo un $22 \%$ de los estudiantes la utilizaron con menor frecuencia. En la tabla 5 se detallan todas las respuestas dadas.

tabla 5. Análisis de uso de la Web App

\begin{tabular}{|l|c|c|}
\hline Frecuencia de visita & Respuestas & Porcentaje \\
\hline A diario & 1 & $11 \%$ \\
\hline $\begin{array}{l}\text { Al menos una vez a la } \\
\text { semana }\end{array}$ & 6 & $67 \%$ \\
\hline Al menos una vez al mes & 1 & $11 \%$ \\
\hline Con menor frecuencia & 1 & $11 \%$ \\
\hline
\end{tabular}

Con respecto al lugar dónde se utilizó, la mayoría de los estudiantes se conectaron bien en su propia casa (44\%) o en algún medio de transporte (33\%):

TABLA 6. LUgar de UtiLIzACIÓn de La Web App

\begin{tabular}{|l|c|c|}
\hline Lugar & Respuestas & Porcentaje \\
\hline En mi casa & 4 & $44 \%$ \\
\hline En un medio de transporte & 3 & $33 \%$ \\
\hline Fuera de casa & 1 & $11 \%$ \\
\hline En el trabajo & 1 & $11 \%$ \\
\hline
\end{tabular}

Y el tiempo medio de la visita se muestra en la tabla 7. Se puede ver que la gran mayoría de las visitas (89\%) son de menos de 30 minutos, siendo siempre menores que 1 hora. 
TABla 7. TIEMPo Medio de Visita de Web ApP

\begin{tabular}{|l|c|c|}
\hline Tiempo medio de visita & Respuestas & Porcentaje \\
\hline Menos de 1 hora & 1 & $11 \%$ \\
\hline Menos de 30 minutos & 3 & $33 \%$ \\
\hline Menos de 15 minutos & 2 & $22 \%$ \\
\hline Menos de 10 minutos & 3 & $33 \%$ \\
\hline
\end{tabular}

Resumiendo este apartado, se puede decir de una manera general la Web App se utilizó una vez por semana, normalmente en casa o en un medio de transporte, utilizándola entre 10 y 30 minutos cada vez.

\subsubsection{Dispositivo más adecuado y ventajas}

Como dispositivo con el que han accedido, según se observa en la tabla 8, fue el Smartphone/Iphone, con un 56\%, a lo que hay que añadir aquellos estudiantes (22\%) que accedieron tanto con éstos como con Tablet/lpad:

TAbla 8. Dispositivo utilizado Web App

\begin{tabular}{|l|c|c|}
\hline Dispositivo utilizado & Respuestas & Porcentaje \\
\hline Smartphone / Iphone & 5 & $56 \%$ \\
\hline Tablet / Ipad & 2 & $22 \%$ \\
\hline Con ambos & 2 & $22 \%$ \\
\hline
\end{tabular}

Con respecto al sistema operativo del dispositivo, según se detalla en la tabla 9, únicamente se consignaron iOS y Android. Android tiene una mayor preponderancia con un $67 \%$ del total, contando los que utilizaron los dos sistemas operativos:

TABLA 9. Sistema OPERATIVO disPositivos

\begin{tabular}{|l|c|c|}
\hline Sistema Operativo & Respuestas & Porcentaje \\
\hline iOS de Apple & 3 & $33 \%$ \\
\hline Android & 5 & $56 \%$ \\
\hline Con ambos & 1 & $11 \%$ \\
\hline
\end{tabular}

Como dispositivo preferido para este tipo de aplicaciones, en la figura 7 se observa que los estudiantes se decantan por Tablet/lpad (56\%) con respecto a los Smartphone/Iphone (33\%): 


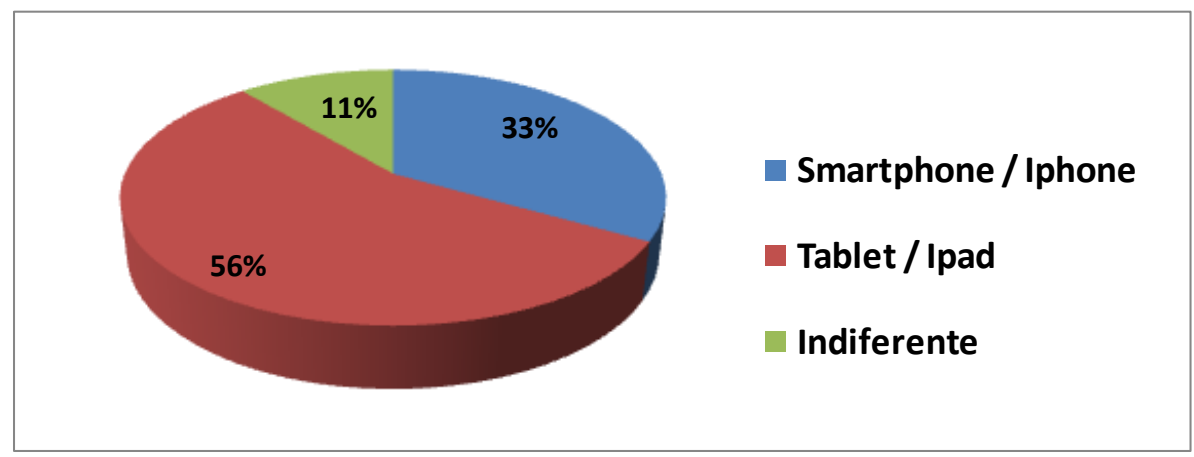

Figura 7. Dispositivo preferido

Las ventajas que percibieron de la Web App frente al Aula de la asignatura fueron:

"Facilidad a la hora de conectarse en cualquier momento sobre todo mientras se viaja en un medio de transporte." "Sobretodo la facilidad de acceso y el diseño adaptado a los smartphones". "Posibilidad de acceder al contenido desde cualquier lugar y cualquier parte, en un determinado momento." "Accesibilidad y movilidad.". "Acceso desde cualquier lugar". "Estudiar desde cualquier sitio en cualquier momento".

A partir de las respuestas precedentes, se puede decir que se accedió principalmente con Smartphone / Iphone, pero como dispositivo preferido para el acceso son las Tablet / Ipad. El tamaño de pantalla de los Tablet / Ipad hace que sean más adecuados para una mejor visualización, pero la mayor movilidad de los Smartphone / Iphone logra que sean estos últimos más utilizados. Como ventajas destacan el poder acceder en cualquier momento y en cualquier sitio, así como el diseño adaptado al dispositivo

\subsubsection{Mejoras propuestas}

Los estudiantes comentaron las siguientes posibles mejoras:

"Más actividades de repaso para realizar". "Chat". "Incluiría una mejora estética y de usabilidad, dando prioridad a la experiencia de usuario para que el uso sea más intuitivo"."Ninguna".

\subsection{Entrevistas}

\subsubsection{Estudiantes de la asignatura}

Se entrevistó a cinco nuevos estudiantes que no habían contestado la encuesta, de los que tres de ellos sí habían utilizado la aplicación. Los dos estudiantes que no la utilizaron comentaron que les parecía una muy buena iniciativa, pero que no habían dispuesto de tiempo suficiente para probarla y ver las posibilidades que tiene. En ambos casos sí que disponían de dispositivos móviles adecuados.

De la conversación tenida con los tres estudiantes que sí la habían utilizado, se extrae lo siguiente:

- Todos los estudiantes la probaron tanto con Smartphone/Iphone como con Tablet/Ipad, utilizando tanto Android como iOS. Los tres estudiantes coincidieron en que Tablet / Ipad era el dispositivo más adecuado, si bien también veían su utilidad en los Smartphones/Iphone. Creían que el tamaño de la pantalla era un factor importante.

- Dos de los tres opinaron que el número de secciones era el adecuado, y otro que 
eran demasiadas, que lo ideal sería 6. Como secciones más útiles, hubo unanimidad de respuestas, y eran los Vídeos y los Casos prácticos. Después de su uso consideraron los tres encuestados que los podcast y el Acceso al Aula Moodle como las secciones menos útiles.

- La usabilidad fue valorada positivamente por todos, destacando su facilidad de uso y navegación intuitiva. En las mejoras propuestas, dos estudiantes creían que sería bueno el incluir más test de autocomprobación y casos prácticos resueltos. El otro estudiante no creía que fueran necesarias nuevas funcionalidades ni mejoras.

- Todos coinciden en afirmar que su mayor uso ha sido en casa, a través de su propia wifi, y que en ocasiones la han utilizado en los medios de transporte.

- Todos ellos piensan que son de utilidad este tipo de aplicaciones, y que su utilización les ha ayudado a afianzar conocimientos en momentos concretos. Coinciden también en que es una buena herramienta complementaria, y que les aporta principalmente rápido acceso a los materiales de la asignatura. Así mismo pensaban que era adecuada tanto para asignaturas teóricas como prácticas, indistintamente.

- Después del uso de la Web App, todos los entrevistados están de acuerdo en que si tuvieran posibilidad de usar este tipo de aplicaciones en otras asignaturas, sin duda, las utilizarían. Creen que el resto de sus compañeros también las utilizarían, aunque no todos. Piensan que entre un $50 \%$ y un $75 \%$ de los estudiantes.

- Todos valoran la experiencia de manera muy positiva, y piensan que ya no solo el futuro, sino el presente de la educación, pasa por complementos de este estilo. La puntuación media que obtuvo fue de 9,33 sobre 10. Las ventajas que detectaron fueron el acceso a los contenidos rápido y sin necesidad de entrar en el Aula Moodle de la asignatura, así como la posibilidad de su uso en cualquier lugar y momento. Como única desventaja se comentó que era necesario tener cobertura $3 G$ o superior, o una conexión wifi, para su uso.

\subsubsection{Estudiantes de otras asignaturas}

Se realizó la entrevista a 5 estudiantes de otras asignaturas de la Facultad, que no habían tenido acceso a ninguna aplicación similar. Probaron la Web App durante 7 días. Sus respuestas pueden resumirse de la siguiente forma:

- Todos los estudiantes probaron tanto con Smartphone/Iphone como con Tablet/lpad. Todos tenían Android en alguno de los dispositivos utilizados, y además también dos de ellos tenían iOS. Cuatros de los cinco estudiantes coincidieron en que Tablet / Ipad era el dispositivo más adecuado, y otro en que era indiferente. De todas formas todos resaltaron que los Smartphone / Iphone también eran adecuados para su uso, y que para algún modulo concreto, como por ejemplo los podcast, era mejor esta última opción.

- Todos coincidieron que el número de secciones era correcto, y que el número deseable debería estar entre 6 y 9 secciones. En el tema de las secciones más útiles hubo bastante variedad en las respuestas, destacando como más útiles los Vídeos y los Casos prácticos, y como menos útiles los Podcast y el Calendario. En todo caso argumentaban que esta respuesta también dependería de la materia concreta para la que estaría desarrollada la Web App.

- Todos los estudiantes, sin excepción, valoraron muy positivamente su facilidad de uso, su rapidez y su navegación intuitiva. Como posibles mejoras y nuevas secciones destaca el que la Web App pudiera poner en contacto a los alumnos 
con el profesor, bien a través del correo electrónico o de algún Foro de respuestas. También algunos pensaron que sería útil que interactuara con el Aula Moodle, y se pudiesen hacer actividades a través de la misma. En ningún caso dijeron de quitar alguna sección, ya que consideraban que siempre habrá algún estudiante que utilice esa sección.

- Como lugares en los que creen que se podría utilizar más están, por este orden: medios de transporte, en cualquier espera que se tenga, así como en segundas residencias o lugares de vacaciones.

- Todos los alumnos estuvieron de acuerdo en que tienen utilidad y ayudan al aprendizaje, ya que facilitan el acceso inmediato a los contenidos de la materia. También afirmaron que consideraban la Web App como una muy buena herramienta complementaria, destacando con respecto al Aula Moodle su inmediatez, el que no necesitan introducir ninguna clave para el acceso, y la movilidad que proporciona. Así mismo pensaban que era adecuada tanto para asignaturas teóricas como prácticas, aunque habría que adaptar las secciones a la idiosincrasia propia de cada asignatura.

- Todos afirmaron que sí que utilizarían este tipo de aplicaciones, y en su mayoría pensaban que el resto de estudiantes también lo harían.

- La valoración general que dieron todos fue muy positiva, pensando que aplicaciones de este estilo serían muy útiles en todas las asignaturas que cursasen. La puntuación media que otorgaron a esta iniciativa fue de 9 sobre 10. Como principales ventajas destacaron su rapidez de acceso a los contenidos, la movilidad que les aportaba, su facilidad de uso, la innovación que representa y que es un perfecto complemento al estudio. Como inconvenientes realmente no hay dos estudiantes que propongan el mismo. El no poder realizar las actividades a través de ella y la poca utilidad del calendario son, quizás, los más significativos.

\subsubsection{Encuesta a profesores}

Se realizó la entrevista a 5 profesores, que previamente probaron la Web App durante 7 días. Las conclusiones extraídas de las conversaciones son las siguientes:

- Todos los profesores habían accedido con Smartphone/lphone, y la mayoría también con Tablet/Ipad, siendo los sistemas operativos tanto Android como iOS. La mayoría opinaba que el dispositivo más adecuado dependía de cada persona y de la situación en que se encontrase. En esta pregunta no hubo una respuesta clara.

- Con respecto al número de secciones todos comentaron que les parecía adecuado, diciendo en algún caso que se podría incluso añadir una sección más y que fueran nueve como máximo. Como secciones más útiles fueron los Vídeos y los Casos prácticos. Como las menos útiles situarían el Calendario y los Podcast, aunque el Calendario también fue situado por alguno de los profesores como una de las secciones más útiles. No hubo unanimidad en las respuestas de los cinco profesores.

- Todas las opiniones al respecto de su usabilidad fueron muy favorables, haciendo hincapié en su rapidez y fácil uso. En las propuestas de nuevas secciones se comentaron varias posibilidades, pero ninguno de los profesores coincidió con otro, salvo en incluir Actividades de Evaluación Continua en la Web App. También dijeron la posibilidad de incluir una sección de Contacto, con correo electrónico y teléfono, para poder contactar con el profesor, la inclusión de un cronograma de la asignatura, y un chat para la comunicación entre los propios estudiantes. 
- Como lugar preferido para su uso todos coincidieron en los medios de transporte, y la mayoría también propuso su uso en cualquier espera que se tenga. Otras opciones que se comentaron fueron para su uso en viajes de varios días, y en casa para el caso de las Tablet/ Ipad.

- Todos le vieron una gran utilidad a este tipo de aplicaciones, pensando que ayuda o facilita el acceso al aprendizaje. Un profesor dijo es una manera de "llevar tu clase contigo siempre". Así mismo creen que es una buena herramienta complementaria, que aporta una mayor rapidez, movilidad y accesibilidad que el Aula Moodle de la asignatura. También consideraron que era útil tanto para asignaturas teóricas como prácticas.

- Todos se mostraron dispuestos a utilizar aplicaciones de este estilo, pero también opinaban que necesitarían un buen soporte técnico. Creen que la mayoría de los estudiantes las utilizarían.

- La valoración general fue muy positiva, haciendo comentarios como que lo consideran una idea innovadora, que facilita el seguimiento de la asignatura y que desarrolla las capacidades TICs de los alumnos. La puntuación media que otorgaron a esta iniciativa fue de 9,2 sobre 10. Como ventajas principales destacaron la rapidez de acceso a los contenidos de la asignatura, la movilidad que proporciona, y que facilita el aprendizaje de los estudiantes. Por el contrario, como desventajas, está que es necesario tener una buena conexión de datos en los dispositivos móviles, que pueda convertirse en un sustitutivo del Aula Moodle, y los efectos negativos en la adquisición de determinadas capacidades de los estudiantes, como creatividad y resolución de problemas, al facilitarle tanto los contenidos.

\section{CONCLUSIONES - DISCUSIÓN DE LOS RESULTADOS}

La gran importancia de aplicar las nuevas tecnologías al aprendizaje universitario, y en concreto al Mobile Learning, es palpable. Existe una gran dificultad debido a los diferentes tipos de dispositivos móviles, con sus distintos tamaños de pantalla y sistemas operativos. Esto implica múltiples desarrollos para crear aplicaciones específicas para cada dispositivo. Para hacer frente a esta problemática, en el presente artículo se propone el uso de aplicaciones web (Web Apps) desarrolladas con Diseño Web Adaptable (Responsive Web Design - RWD). Este diseño aporta grandes ventajas sobre las Apps creadas para un determinado sistema operativo, evitando su descarga, y que funcionan independientemente del dispositivo utilizado. Esto proporciona que sólo sea necesario el desarrollo de un site único, logrando que los contenidos (texto, imágenes, vídeos, etc.) se adapten automáticamente al tamaño de la pantalla. La actualización de los contenidos se realiza de una forma muy sencilla mediante el almacenamiento en la nube.

Se observa que la prueba piloto realizada en la asignatura de Dirección de la producción ha sido un éxito, aportando a los estudiantes un entorno ideal para el Mobile Learning Multidispositivo como ayuda al estudio y aprendizaje de la asignatura. Tanto los resultados de la encuesta, como de las entrevistas realizadas, muestran que este tipo de iniciativas son válidas para los dispositivos móviles más utilizados en la actualidad, como son los Smartphone/Iphone, como para Tablet/Ipad. Como dispositivo preferido están las Tablet e lpad, debido a su mayor tamaño de pantalla. No obstante esa ventaja para la visualización de los contenidos, es una desventaja para la movilidad, por lo que es importante que esté accesible también a los Smartphones e Iphone. Los contenidos asociados a la Web App son todos bien valorados, siendo especialmente importantes las secciones de vídeos y de casos 
prácticos resueltos. Se resaltan como grandes ventajas su fácil uso, el acceso rápido, inmediato, en cualquier lugar y momento. Se opina que los lugares ideales para su uso son los medios de transporte, cualquier tiempo de espera que se tenga, así como para viajes. También hay que resaltar el uso que se hace en el propio hogar de las Tablet/lpad, mediante wifi. Como principal desventaja, se cita que es necesario tener acceso a una red de datos para su uso. La valoración dada, tanto por estudiantes, como por profesores es realmente muy positiva, siendo siempre igual o superior a 9 sobre 10.

En base a todo lo expuesto anteriormente, creemos que el desarrollo de Web Apps para las diferentes asignaturas en un entorno universitario es una gran solución para el Mobile Learning Multidevice.

\section{BIBLIOGRAFÍA}

ABACHI, H. R., \& MUHAMMAD, G. (2014). The impact of m-learning technology on students and educators. Computers in Human Behavior, 30, 491-496.

ADAM, D., KIOUTSIOUKI, D., KARAKOSTAS, A., \& DEMETRIADIS, S. N. (2014). Do Your Students Get It? Quiz It! The Android Classroom Response System. In Advanced Learning Technologies (ICALT), 2014 IEEE 14th International Conference on (pp. 168-170).

AMER, H., \& IBRAHIM, W. (2014). Using the iPad as a pedagogical tool to enhance the learning experince for novice programing students. In Global Engineering Education Conference (EDUCON), 2014 IEEE (pp. 178-183).

BATURAY, M. H., \& BIRTANE, M. (2013). Responsive Web Design: A New Type of Design for Web-based Instructional Content. Procedia-Social and Behavioral Sciences, 106, 2275-2279.

BIDIN, S., \& ZIDEN, A. A. (2013). Adoption and Application of Mobile Learning in the Education Industry. Procedia-Social and Behavioral Sciences, 90, 720-729.

BRAZUELO GRUND, F., \& CACHEIRO GONZÁLEZ, M. L. (2010). Diseño de páginas web educativas para teléfonos móviles. Edutec: Revista electrónica de tecnología educativa, (32), 2.

DE LA PEÑA, F. D. \& BURGOS, M. C. (2015). Modelo práctico de aplicación (app) para dispositivo móvil en las asignaturas universitarias de enseñanza a distancia. Experiencia con Android para la asignatura Dirección de Operaciones. EDUTEC, Revista Electrónica de Tecnología Educativa, 51.

GIEMZA, A., BOLLEN, L., JANSEN, M., \& HOPPE, H. U. (2012). An architecture for supporting heterogeneous multi-device learning environments. In Wireless, Mobile and Ubiquitous Technology in Education (WMUTE), 2012 IEEE Seventh International Conference (pp. 67-71).

GIKAS, J., \& GRANT, M. M. (2013). Mobile Computing Devices in Higher Education: Student Perspectives on Learning with Cellphones, Smartphones \& Social Media. Internet and Higher Education 19 (2013) 18-26.and Higher Education.

GLASSMAN, N. R., \& SHEN, P. (2014). One Site Fits All: Responsive Web Design. Journal of Electronic Resources in Medical Libraries, 11 (2), 78-90.

GORDILLO, A., BARRA, E., GALLEGO, D., \& QUEMADA, J. (2013). An online e-Learning authoring tool to create interactive multi-device learning objects using eInfrastructure resources. In Frontiers in Education Conference, 2013 IEEE (pp. 1914-1920). 
HARGIS, J., CAVANAUGH, C., KAMALI, T., \& SOTO, M. (2014). A federal higher education iPad mobile learning initiative: triangulation of data to determine early effectiveness. Innovative Higher Education, 39(1), 45-57.

ISABWE, G. M. N., REICHERT, F., \& CARLSEN, M. (2013). Rethinking practices of assessment for learning: Tablet technology supported assessment for learning mathematics. In Teaching, Assessment and Learning for Engineering (TALE), 2013 IEEE International Conference on (pp. 155-159). IEEE.

KONG, L. (2013). The Development of Mobile Learning System Based on the Android Platform. In Proceedings of the 2012 International Conference of Modern Computer Science and Applications (pp. 701-706). Springer Berlin Heidelberg.

KOUSAR, M. N., \& SCHOLAR, P. D. (2013) Feasibility study of mobile learning in distance education. Work paper of Distance, Non-Formal and Continuing Education Department. Allama labal Open University, Islamabad.

MAMAT, K., \& AZMAT, F. (2013). Mobile Learning Application for Basic Router and Switch Configuration on Android Platform. Procedia-Social and Behavioral Sciences, 90, 235-244.

MARTIN, F., \& ERTZBERGER, J. (2013). Here and Now Mobile Learning: An Experimental Study on the Use of Mobile Technology. Computers \& Education. Volume 68, October 2013, Pages 76-85.

MOLNAR, G. (2012). New learning spaces? M-learning's, in particular the iPad's potentials in education. In Interactive Collaborative Learning (ICL), 2012 15th International Conference on (pp. 1-5).

OBERER, B., \& ERKOLLAR, A. (2013). Mobile Learning in Higher Education: A Marketing Course Design Project in Austria. Procedia-Social and Behavioral Sciences, 93, 2125-2129.

OZUORCUN, N. C., \& TABAK, F. (2012). Is M-learning Versus E-learning or are They Supporting Each Other?. Procedia-Social and Behavioral Sciences, 46, 299-305.

SARRAB, M., ELGAMEL, L., \& ALDABBAS, H. (2012). Mobile learning (m-learning) and educational environments. International Journal of Distributed and Parallel Systems, 3(4), 31-38.

SETIABUDI, D. H., \& TJAHYANA, L. J. (2013). Mobile Learning Application Based On Hybrid Mobile Application Technology Running On Android Smartphone and Blackberry (Doctoral dissertation, Petra Christian University).

STEAD, G. (2014). Open Formats for Mobile Learning. Chapter 8 of Increasing Access through Mobile Learning, 99-112.

VALERO, C. C., REDONDO, M. R., \& PALACíN, A. S. (2012). Tendencias actuales en el uso de dispositivos móviles en educación. La Educación Digital Magazine, 147, 1-21.

YANG, H. C., \& WANG, W. Y. (2011). Facilitating Academic Service-Learning with Android-Based Applications and Ubiquitous Computing Environment. In UbiMedia Computing (U-Media), 2011 4th International Conference on (pp. 191196). 
F. David de la Peña, M. Concepción Burgos, M. Antonia Simón.

\section{Webgrafía}

Estudio DIGITAL, SOCIAL \% MOBILE IN 2015: http://wearesocial.net/tag/sdmw/

Estudio KPCB Internet Trends, 2014: www.kpcb.com/internet-trends

$\checkmark$ Estudio Anual IAB Spain Mobile Marketing, 2014: www.iabspain.net/mobilemarketing/

\section{ANEXOS}

\subsection{Encuesta realizada a los alumnos}

1. ¿̇Has utilizado la aplicación? Sí / No

2. ¿Con qué dispositivo has accedido a la aplicación?

Smartphone / Iphone

Tablet / Ipad

Con ambos

Otro:

3. ¿Qué sistema operativo tienen tus dispositivos móviles?

Android

iOS de Apple

Tanto Android como iOS de Apple

Otro:

4. ¿Cuántas veces has visitado la aplicación de la asignatura?

A diario

Al menos una vez a la semana

Al menos una vez al mes

Con menor frecuencia

Nunca

5. Puntúa de 1 a 5 la utilidad de la aplicación (estudio, repaso, accesible desde cualquier lugar, ...)

6. Puntúa de 1 (menos) a 5 (más) la utilidad de cada de las secciones:

Calendario

Vídeos Teoría

Vídeos Casos

Sesiones Virtuales

Unidades

Casos prácticos

Podcast

Test

Acceso a Aula Udima 
7. Puntúa de 1 a 5 la usabilidad de la aplicación (navegación intuitiva, rapidez, disposición de las secciones, facilidad de uso).

8. Mi seguimiento de la asignatura ha mejorado después de descargar la aplicación (Puntúa de 1 a 5).

9. Habitualmente, ¿̇dónde se encontraba usted cuando entraba en la aplicación?

En mi casa

En el trabajo

En un medio de transporte

Lugares de ocio

Otro:

10. Habitualmente ¿̇cuánto tiempo de media ha utilizado la aplicación cada vez que ha accedido a ella?
Menos de 5 minutos
Menos de 10 minutos
Menos de 15 minutos
Menos de 30 minutos
Menos de 1 hora
Más de 1 hora

11. Creo que la existencia de aplicaciones en las asignaturas mejorarían mi aprendizaje y estaría dispuesto a descargármelas (Puntúa de 1 a 5).

12. ¿Cuál crees que es el dispositivo móvil más adecuado para visualizar esta aplicación?

\author{
Smartphone / Iphone \\ Tablet / Ipad \\ Indiferente \\ Otro:
}

13. ¿Qué ventajas crees que te da la aplicación con respecto al Aula de la asignatura?

14. ¿¿Qué mejoras incluirías en la aplicación?

En caso de no haber utilizado la aplicación:

2.¿̇Cuál es el motivo de que no hayas utilizado la aplicación?

Tengo Smartphone / Iphone pero no la iba a utilizar

Tengo Tablet/ Ipad pero no la iba a utilizar

No dispongo de dispositivos móviles

Otro: 


\subsection{Guión de las entrevistas realizadas}

- ¿̇Con qué dispositivos móviles has accedido a la aplicación? ¿̇ué sistema operativo tienen?

- ¿̇Cuál crees que es el dispositivo móvil más adecuado para visualizar esta aplicación?

- ¿ ¿Qué opinas del número de secciones? (Muchas / Pocas)

- ¿ ¿Qué secciones crees que son las más útiles? ¿Y menos? Nombra dos o tres de cada una.

- ¿ ¿Cómo valoras la usabilidad de la Web App?

- ¿ ¿Qué mejoras incluirías en la aplicación? ¿ Qué secciones añadirías / quitarías? $\dot{\imath}$ e te ocurre alguna nueva funcionalidad?

- ¿ ¿Cuál sería el lugar/es donde crees que su uso es el más adecuado?

- ¿ ¿Crees qué tienen utilidad este tipo de aplicaciones? ¿̇Ayudan al aprendizaje del estudiante?

- ¿ ¿Crees que es una buena herramienta complementaria de las asignaturas? ¿̇Te aporta algo distinto del Aula de la asignatura?

- ¿ ¿ ¿Crees que sería útil para todo tipo de asignaturas? (Teóricas /Prácticas)

- Como profesor/estudiante, ¿utilizarías aplicaciones de este estilo? ¿̇Crees que los otros estudiantes la utilizarían?

- ¿̇Podrías dar una valoración general, tanto cualitativa como cuantitativa de este tipo de aplicaciones(de 0 a 10)? ¿ ¿Qué ventajas e inconvenientes crees que tiene? 